\title{
Estudio de la asociación entre la discriminación laboral, la salud y el uso de servicios sanitarios en Andalucia:Una aplicación de propensity score matching con muestras pequeñas ${ }^{1}$
}

\author{
Study of the association between labour discrimination, \\ health and use of health services in Andalucia: an \\ application of propensity score matching with small \\ samples
}

\author{
JaVIer Álvarez-GÁLVEZ \\ Universidad de Cádiz \\ javier.alvarezgalvez@uca.es (ESPAÑA) \\ Alvaro Suárez-Vergne* \\ Universidad Complutense de Madrid \\ alvarsuar@ucm.es (ESPAÑA)
}

Recibido: 03.01.2019

Aceptado: 28.05.2020

\section{RESUMEN}

El presente estudio tiene como objetivo explorar la asociación entre discriminación laboral y salud en Andalucía (España). Este estudio se basa en una muestra de 1200 individuos. El indicador de discriminación laboral fue diseñado a través de una pregunta abierta que se refería a otros tipos de discriminación. Aunque la categoría discriminación laboral no se incluyó inicialmente en el cuestionario, el $65 \%$ de los participantes se consideraron sujetos a discriminación laboral mientras que solo el 35\% informaron otras causas habituales de discriminación. Las medidas de propensión muestran que las personas que reportan discriminación laboral presentan un mayor uso de servicios de salud primaria,

\footnotetext{
${ }^{1}$ Esta investigación se ha realizado dentro del proyecto IMPACT-A, 'Determinantes socioeconómicos de salud en Andalucía: estudio comparado del impacto de la crisis económica en la salud de los andaluces' (PRY120/14) subvencionado por la Fundación Pública Centro de Estudios Andaluces.

${ }^{*}$ Beneficiario de una ayuda para la formación del profesorado universitario (FPU-2017)
}

EMPIRIA. Revista de Metodología de Ciencias Sociales. N. ${ }^{\circ} 48$ septiembre-diciembre, pp. 147-166.

ISSN: 1139-5737, DOI/ empiria..48.2020.28074 
un resultado que podría estar relacionado con la mayor prevalencia de trastornos depresivos en grupos socioeconómicamente desfavorecidos. Aunque se necesita profundizar en esta materia, la orientación futura de las políticas sociales y de salud de la UE deberían incluir como objetivo la reducción del impacto social del desempleo y la precariedad laboral a fin de reducir la discriminación y promover la equidad en salud.

\title{
PALABRAS CLAVE
}

Disscriminación laboral; determinantes sociales de la salud; uso de servicios de salud; calidad de vida; propensity score matching.

\begin{abstract}
The present study aims to explore the association between employment discrimination and health in Andalusia (Spain). This study is based on a sample of 1200 individuals. The employment discrimination indicator was designed is through the results of an open-ended question that refers to other types of discrimination. Although the category 'employment discrimination' were not initially included in the questionnaire, $65 \%$ of participants perceived themselves as subject to employment discrimination while only $35 \%$ reported other usual causes of discrimination. Employment discrimination has been found similarly associated to poor health outcomes. Propensity scores show people reporting employment discrimination present a higher use of primary health services, a result that might be related with the higher prevalence of depressive disorders of socioeconomically disadvantaged groups. Although additional research is needed, the future orientation of EU social and health policies should aim to reduce the social impact of unemployment and insecure jobs in order to reduce discrimination and promote health equity.
\end{abstract}

\section{KEY WORDS}

Employment discrimination; social determinants of health; health services use; quality of life; propensity score matching.

\section{INTRODUCCIÓN}

Los procesos de discriminación tienen efectos sobre nuestra salud física y mental (Paradies, 2006; Alvarez-Galvez y Salvador-Carulla, 2013). Recientes evidencias demuestran que las personas que se sienten socialmente discriminadas presentan una mayor propensión hacia el desarrollo de problemas de salud 
mental como el estrés, la ansiedad, las fobias, la depresión o el desarrollo de conductas de riesgo para la salud (Pascoe y Smart, 2009). Del mismo modo, los problemas psicológicos vinculados al sentimiento de discriminación también pueden derivar en problemas salud física. Niveles de estrés continuados pueden dar lugar a problemas de hipertensión o alteraciones del aparato digestivo (Williams y Mohamed, 2009). El sentimiento de discriminación puede aumentar la predisposición individual hacia el consumo de drogas y, en los casos más graves, empujar al suicidio como una forma de escapar del rechazo y el aislamiento social (Pompili, Mancinelli y Tatarelli, 2003; Miller, Mazza y Ecker, 2009).

Una gran parte de la literatura científica centrada en el estudio del impacto de los procesos de discriminación sobre la salud de la población ha destacado la influencia de la discriminación racial o étnica en los resultados de salud mental y física (Paradies, 2006; Kim y Williams, 2012), pero factores como el género, orientación sexual, idioma, religiosidad, nacionalidad, clase social o discapacidad también se han encontrado asociados a problemas de salud (Alvarez-Galvez, 2016). Sin embargo, a pesar de que la relación entre discriminación y salud ha sido ampliamente estudiada desde diferentes perspectivas, la literatura del ámbito de las ciencias de la salud ha prestado menos atención a los efectos de la discriminación laboral sobre la salud de aquellos individuos que sufren el desempleo de larga duración, que tienen trabajos inseguros o precarios, que ocupan puestos de trabajos irregulares o se dedican a labores domésticas (Ferdinand, Paradies, Perry y Kelaher; 2014). Pese a ello, diversos estudios señalan que la precariedad económica, que podría asociarse con situaciones de discriminación laboral, constituye un factor que influye negativamente sobre la salud, estando relacionada una baja posición socioeconómica con problemas cardiovasculares (Boylan, Cundiff y Matthews, 2018), con el desarrollo de trastornos mentales en menores de edad (Reiss, 2013) y con una reducción de la esperanza de la vida (Stringhini et. al, 2017).

La discriminación laboral es una forma particular de discriminación en el acceso al trabajo y/o vinculada las condiciones laborales, que se da en base a características personales como la raza, el género, la religión, la nacionalidad, la discapacidad física o mental, la edad, la orientación sexual o la identidad de género (Chung, 2001; Roehling, Roehling y Pichler, 2007; Gayman, 2013. Las diferencias de ingresos entre hombres y mujeres dentro de un mismo centro de trabajo y los problemas de acceso al mundo laboral de personas con discapacidad son algunos de los ejemplos más comunes de discriminación laboral, una forma de discriminación que puede haber aumentado en los últimos años a causa de la reciente crisis económica.

Pese a que a que los atributos personales que desencadenan las situaciones de discriminación laboral pueden ser muy variados (género, nacionalidad, discapacidad etc.) podemos considerar que esta constituye un tipo concreto de discriminación, puesto que recoge un conjunto de acciones desfavorables que afectan a la situación laboral de un determinado sujeto. En este sentido, la definición de discriminación laboral no está ligada a un atributo personal concreto, sino a un contexto social, el mercado laboral. Estudiar la discriminación laboral conside- 
rando a esta como una categoría específica de análisis es de gran utilidad para aproximarse a dinámicas sociales negativas que pueden influir en situaciones de exclusión económica, siendo el grado de integración laboral uno de los componentes fundamentales de dicho concepto (Lightman y Good, 2018).

No obstante, debe tenerse en cuenta que los mismos factores que influyen en las situaciones de discriminación laboral también pueden originar dinámicas discriminatorias en otros ámbitos que van más allá del mercado de trabajo. Esto no resta validez a la constitución de la discriminación laboral como una categoría susceptible de análisis, pero si hace necesario profundizar en qué colectivos son más proclives a experimentar este tipo de discriminación con objeto de contrastar posteriormente si estos grupos pueden verse afectados por acciones desfavorables en otros ámbitos, lo que incrementaría su vulnerabilidad.

A priori, puede intuirse que los colectivos sobre los que pesan estereotipos desfavorecedores como es el caso de la población inmigrante (Santamaría, 2002), las mujeres (Schur, 1984) o los sujetos con trastornos mentales (Corrigan y Watson, 2002), son más proclives a experimentar situaciones de discriminación laboral, al relacionarse algunas de las concepciones negativas que afectan a estos grupos con las capacidades laborales. Un ejemplo sería el caso de los sujetos con trastornos mentales, en ocasiones percibidos por los empleadores como incompetentes a la hora de realizar diferentes tareas (Corrigan y Watson, 2002), lo que reduce sus posibilidades de acceder a un empleo. Asimismo debe tenerse en cuenta el papel del poder, derivado de un mayor/menor estatus social, en el sentido de que son los colectivos con menor estatus los que menos recursos tienen para hacer frente a las situaciones de discriminación, las cuales además cronifican su posición inferior de poder manteniendo así el orden social y económico (Link y Phelan, 2001; 2014, Link, Phelan y Dovidio, 2008). Algunos ejemplos de ello serían la explotación laboral de colectivos inmigrantes (IOE, 2000) o la precarización del empleo femenino (Alcañiz, 2015).

Todo ello apunta a que son los colectivos que parten de una situación social desventajosa y que ya son discriminados en otras áreas (mujeres, inmigración, mayores, discapacitados, etc.) los que presentan una mayor facilidad para sufrir discriminación laboral y, por consiguiente, aquellos que en mayor medida sufrirían el impacto de las desigualdades sociales en salud (Álvarez-Gálvez y Salvador-Carulla, 2013). En definitiva, las dinámicas descritas podrían contribuir a la ampliación de la brecha socio-sanitaria entre la población general y los grupos sociales más desfavorecidos que, en paralelo, se encuentran más expuestos a los procesos de discriminación. Más aún tras la crisis económica iniciada en el año 2008 (Álvarez-Gálvez et al. 2019), la cual ha tenido especial efecto en diversos colectivos como, por ejemplo, los jóvenes de clase baja (Mínguez, 2015), la población inmigrante (Carpio y García-Serrano, 2015), los mayores y los sujetos con alguna discapacidad (Alcover et al., 2017), o las mujeres (Muñoz y Modroño, 2012). La crisis financiera y las políticas de austeridad han contribuido a la ampliación de las desigualdades socioeconómicas en las diferentes regiones de la geografía española. Mediante la aplicación de diferentes técnicas de análisis de datos, el presente estudio tiene como objetivo explorar la asociación entre la 
experiencia de la discriminación laboral y la salud tomando la región de Andalucía como caso de estudio.

\section{MÉTODO}

\subsection{Muestras y variables del estudio}

Este trabajo se basa en una muestra de 1200 personas del proyecto IMPACT-A, cuyo trabajo de campo se llevó a cabo entre los meses de marzo y abril del año 2016. Los participantes del estudio fueron seleccionados al azar siguiendo un proceso de muestreo estratificado en función de la provincia y el tamaño del hábitat. La población objetivo de esta encuesta incluyó a personas mayores de 18 años residentes en Andalucía. La encuesta final está compuesta por personas de las ocho provincias andaluzas: Almería, Cádiz, Córdoba, Granada, Huelva, Jaén, Málaga y Sevilla.

Para comparar la asociación entre la discriminación laboral y la salud se analizaron diferentes variables dependientes: (1) índice de calidad de vida (SF-12); (2) salud auto-percibida donde 1 significa 'Muy mala', 2 'Mala', 3 'Aceptable', 4 'Buena' y 5 'Muy buena'; (3) índice de masa corporal (IMC); (4) felicidad, donde 0 indicaría sentirse 'Totalmente infeliz' y 10 'Totalmente feliz'; y finalmente (6) el uso de servicios de salud (atención primaria, urgencias, ingresos hospitalarios y servicios especializados). Por otro lado, el indicador de discriminación auto-percibida se extrajo de la siguiente pregunta del cuestionario: ‘¿Por qué motivo se siente principalmente discriminado?' Se incluyeron diez respuestas posibles: (1) raza o color, (2) nacionalidad, (3) religión, (4) edad, (5) sexo, (6) sexual, (7) discapacidad, (8) educación, (9) clase social y (10) otros motivos. El indicador de discriminación laboral se desarrolló a través de los resultados de una pregunta abierta de esta última categoría que incluía otras razones.

\subsection{Diseño del indicador de discriminación laboral}

Aunque la discriminación laboral no era una categoría de análisis que se concretase en el cuestionario del estudio, los resultados exploratorios de los datos de la encuesta mostraron que, en Andalucía, el tipo de discriminación más prevalente era 'otro', no comúnmente referido en la literatura especializada. El $65 \%$ de los andaluces que indicaban sentirse discriminado declaró haber sido discriminado por otros motivos que, en su mayoría, estaban relacionados con problemas económicos y laborales asociados a la crisis financiera (figura 1) una serie de motivos que pueden integrarse bajo la definición de discriminación laboral desarrollada en el apartado anterior.

Entre los principales motivos de discriminación se encontraron los siguientes: (1) debido a enfermedad y/o discapacidad no puede trabajar, (2) dificultades 
y/o problemas para encontrar trabajo, (3) por ser una mujer en edad fértil que no puede encontrar un trabajo, (4) porque no tienen una pensión adecuada en relación a los años trabajados, (5) debido a la falta de oportunidades para grupos jóvenes, (6) por ser un/a desempleado/a mayor de 50 años, (7) por que le persona se siente demasiado mayor como para conseguir un trabajo, (8) porque es pensionista o retirado/a, (9) porque el trabajo que hace no es valorado, esto es, no se percibe como una persona valorada en la actividad laboral que desempeña, (10) por haber sido despedida después de una baja por enfermedad, (11) por tener una categoría laboral de baja calificación en relación a la educación que tiene, (12) por haber perdido los derechos laborales anteriores, (13) por ser una persona desempleada joven, (14) por la falta de oportunidades laborales para su colectivo, (15) porque no encuentra trabajo por ser viejo, (16) porque no tiene acceso al mercado laboral (no encuentra trabajo), (17) por haber sido retirado del mundo laboral siendo joven debido a una enfermedad y/o incapacidad, (18) por ser un/a pensionista con una pensión baja, (19) por tener un trabajo precario con una remuneración baja, (20) por ser un/a parado/a de larga duración, (21) por ser un trabajador autónomo.

Como se puede apreciar, pese a que en las respuestas se hacen referencias a diferentes características personales, todas ellas tienen el denominador común de derivar en la recepción de acciones desfavorables en el marco del mercado laboral. La discriminación laboral se codificó manualmente a partir de estas respuestas abiertas para estos otros tipos de discriminación que hacían referencia a problemas de acceso al trabajo y a situaciones económicas problemáticas asociadas a la falta de empleo o al trabajo precario. La fiabilidad del proceso de codificación, que fue llevado a cabo por dos colaboradores del equipo de investigación, se determinó mediante la prueba alfa de Cronbach. Los resultados de esta prueba pusieron de manifiesto la consistencia del nuevo indicador de discriminación laboral $(\alpha=0.97)$, evidenciando, como se expone anteriormente, la pertinencia de considerar la discriminación laboral como un tipo particular de discriminación. 
Figura 1. Principales tipos de discriminación entre los entrevistados que reportaban algún tipo de rechazo

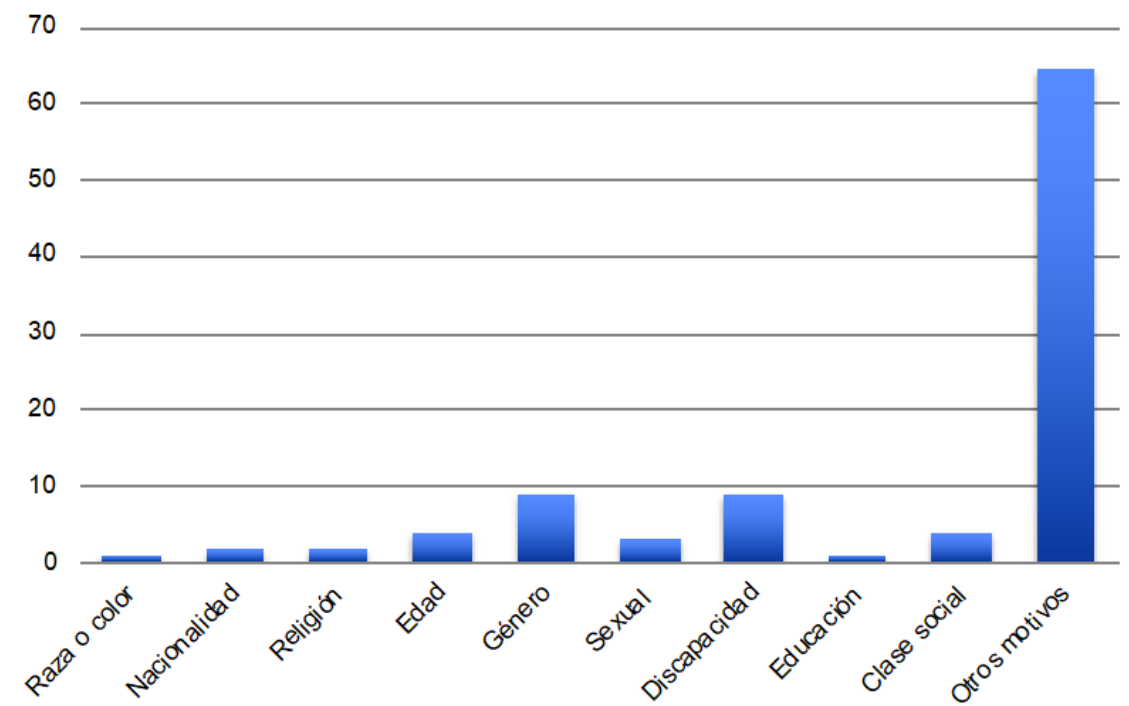

Fuente: Elaboración propia a partir de los datos del Proyecto ANONIMIZADO

\subsection{Análisis estadístico}

Para las variables de salud y los indicadores de calidad de vida, la asociación se estudiaría a partir de modelos ANOVA, mientras que el estudio de las diferencias en el uso de servicios de salud se analizaría mediante tablas de contingencia que serían empleadas para describir las diferencias en las proporciones. Para el análisis bivariado se utilizó el test de asociación de $\mathrm{Chi}^{2}$ y el test exacto de Fischer para medir la asociación entre algunas variables. Los datos se analizaron utilizando el software estadístico Stata v.14.0.

\subsection{Propensity score matching}

Para validar las diferencias detectadas a partir de las técnicas anteriores se realizó un análisis de Propensity Score Matching (PSM) para estimar la asociación entre la discriminación laboral frente a otros tipos y las diferentes variables de salud y bienestar, así como también para las de uso de servicios de sanitarios (atención primaria, especialistas, ingresos hospitalarios, y urgencias) (Austin, 2011). Mediante el uso de la técnica PSM se trataría de medir el efecto específico de la discriminación laboral en relación a los tipos comúnmente analizados 
en la literatura (tomados en su conjunto), y comparar las divisiones específicas en los resultados para las variables analizadas entre el conjunto de provincias andaluzas. Las puntuaciones de propensión se calcularon para cada sujeto y se utilizaron para crear pares de grupos comparables en función de los factores de pronóstico estadísticamente significativos en la regresión logística, así se compararía el efecto de la "discriminación laboral" como una forma mixta de discriminación frente a "otros tipos" (puros) de rechazo. Usando este indicador binario de discriminación laboral se evaluaron las divisiones en la salud y el bienestar de los ciudadanos andaluces, así como las diferencias en el uso de servicios de salud.

El empleo de esta técnica analítica presenta la ventaja de que posibilita el correcto equilibrado de la distribución de los factores de confusión observados, así como la eliminación de posibles sesgos que pudieran surgir en relación al proceso de selección de unidades de análisis o al conjunto de predictores empleados (Austin, 2011). A diferencia de las técnicas multivariadas tradicionales, el PSM puede reducir los errores en la estimación del efecto de los factores de confusión en el resultado, al tiempo que permite la evaluación independiente del balance de las covariables observadas entre los grupos que se comparan (Cepeda et al., 2003). En definitiva, teniendo en cuenta que el presente estudio se basa en el análisis de una muestra pequeña de individuos, con la aplicación de la técnica PSM se verificó la fiabilidad de los resultados obtenidos mediante las técnicas anteriores para la medición del efecto de la discriminación laboral sobre la salud y el uso de servicios sanitarios frente a otras formas de discriminación.

\section{RESULTADOS}

Se ha encontrado que un $8,4 \%$ de los entrevistados $(\mathrm{N}=101)$ se siente parte de un grupo discriminado en este país, de los cuales un 5,5\% se auto-perciben como laboralmente discriminados. El 2,9\% restante se sienten discriminados por razones de color o raza, nacionalidad, religión, edad, género, orientación sexual, discapacidad, nivel educativo o clase social.

En función del sexo se aprecia que los hombres declaran sufrir discriminación laboral en mayor medida que las mujeres, particularmente, se observa que son los grupos en edad laboral los que presentan porcentajes más elevados de discriminación laboral, aunque no se aprecian diferencias estadísticamente significativas con respecto a otras formas comunes de discriminación. Los grupos de ingresos más bajos (C1-C2) o en situación de desempleo son los que más discriminados se sienten por motivos laborales y/o económicos (tabla 1). 
Tabla 1. Comparación del perfil socio-demográfico de los entrevistados en función de la percepción de discriminación (discriminación laboral vs. otros tipos de discriminación).

\begin{tabular}{|c|c|c|c|c|c|}
\hline Variable & Categoría & $\begin{array}{c}\text { No discrimina- } \\
\text { do. }\end{array}$ & $\begin{array}{l}\text { Discrimina- } \\
\text { ción laboral }\end{array}$ & $\begin{array}{l}\text { Otros tipos de } \\
\text { discriminación }\end{array}$ & Sig. \\
\hline \multirow{2}{*}{ Sexo } & Hombre & $90.48 \%$ & $7.14 \%$ & $2.38 \%$ & \multirow{2}{*}{0.015} \\
\hline & Mujer & $92.97 \%$ & $3.59 \%$ & $3.43 \%$ & \\
\hline \multirow{7}{*}{$\begin{array}{l}\text { Grupo de } \\
\text { edad }\end{array}$} & $15-24$ & $96.00 \%$ & $2.00 \%$ & $2.00 \%$ & \multirow{7}{*}{0.716} \\
\hline & $25-34$ & $91.37 \%$ & $4.32 \%$ & $4.32 \%$ & \\
\hline & $35-44$ & $91.29 \%$ & $6.22 \%$ & $2.49 \%$ & \\
\hline & $45-54$ & $90.99 \%$ & $5.86 \%$ & $3.15 \%$ & \\
\hline & $55-64$ & $89.35 \%$ & $7.69 \%$ & $2.96 \%$ & \\
\hline & $65-74$ & $93.08 \%$ & $4.62 \%$ & $2.31 \%$ & \\
\hline & $75+$ & $95.45 \%$ & $3.64 \%$ & $0.91 \%$ & \\
\hline \multirow{4}{*}{$\begin{array}{l}\text { Nivel de } \\
\text { estudios }\end{array}$} & Sin estudios & $95.65 \%$ & $1.09 \%$ & $3.26 \%$ & \multirow{4}{*}{0.299} \\
\hline & Primaria & $90.75 \%$ & $6.57 \%$ & $2.69 \%$ & \\
\hline & Secundaria & $90.81 \%$ & $5.14 \%$ & $4.05 \%$ & \\
\hline & Universidad & $92.50 \%$ & $5.50 \%$ & $2.00 \%$ & \\
\hline \multirow{4}{*}{$\begin{array}{l}\text { Ingresos } \\
\text { (cuartiles) }\end{array}$} & $\mathrm{C} 1$ & $87.83 \%$ & $7.89 \%$ & $4.28 \%$ & \multirow{4}{*}{0.001} \\
\hline & $\mathrm{C} 2$ & $88.79 \%$ & $8.19 \%$ & $3.02 \%$ & \\
\hline & $\mathrm{C} 3$ & $95.58 \%$ & $2.86 \%$ & $1.56 \%$ & \\
\hline & $\mathrm{C} 4$ & $97.30 \%$ & $0.68 \%$ & $2.03 \%$ & \\
\hline \multirow{2}{*}{$\begin{array}{l}\text { Situación } \\
\text { laboral }\end{array}$} & Trabaja & $94.32 \%$ & $3.10 \%$ & $2.58 \%$ & \multirow[b]{2}{*}{0.001} \\
\hline & $\begin{array}{c}\text { Desemplea- } \\
\text { do }\end{array}$ & $81.25 \%$ & $13.02 \%$ & $5.73 \%$ & \\
\hline
\end{tabular}




\begin{tabular}{cccccc} 
Variable & Categoría & $\begin{array}{c}\text { No discrimina- } \\
\text { do. }\end{array}$ & $\begin{array}{c}\text { Discrimina- } \\
\text { ción laboral }\end{array}$ & $\begin{array}{c}\text { Otros tipos de } \\
\text { discriminación }\end{array}$ & Sig. \\
\hline \multirow{6}{*}{ Provincia } & Almería & $92.67 \%$ & $4.67 \%$ & $2.67 \%$ & \\
& Cádiz & $92.67 \%$ & $5.33 \%$ & $2.00 \%$ & \\
& Córdoba & $89.33 \%$ & $5.33 \%$ & $5.33 \%$ & \\
& Granada & $93.33 \%$ & $5.33 \%$ & $1.33 \%$ & 0.200 \\
& Huelva & $93.33 \%$ & $3.33 \%$ & $3.33 \%$ & \\
& Jaén & $87.33 \%$ & $10.67 \%$ & $2.00 \%$ & \\
& Málaga & $91.33 \%$ & $4.00 \%$ & $4.67 \%$ & \\
\hline
\end{tabular}

Los resultados confirman la idea de que la discriminación laboral se encuentra íntimamente ligada a la precariedad económica, siendo los sujetos con bajos ingresos y los que se encuentran en situación de desempleo los que declararan más situaciones de discriminación laboral.

Al comparar la discriminación laboral con respecto a otros tipos comunes de discriminación (raza, género, religión, nacionalidad, etc.) mediante los modelos ANOVA (figura 2), se evidencia el mismo efecto negativo para la salud y la calidad de vida $(\mathrm{p}<0.05)$. Las personas discriminadas, con independencia del motivo de rechazo, presentan una peor calidad de vida, menor grado de felicidad y una peor salud auto-percibida que las personas que no se sienten socialmente excluidas. Las personas discriminadas por motivos laborales presentan un mayor IMC que aquellos que no se sienten discriminados o se encuentran discriminados por otros motivos $(\mathrm{F}=3.68, \mathrm{p}<0.05)$. 
Figura 2: ANOVA para variables de salud y bienestar según tipo de discriminación percibida

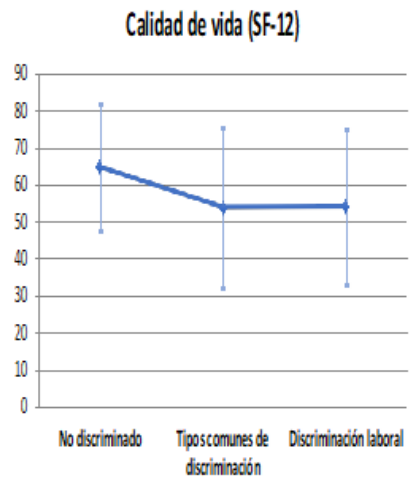

IMC

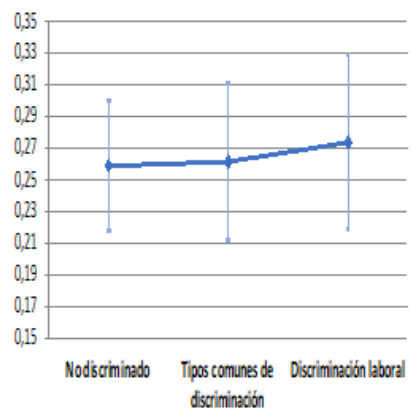

Felididad

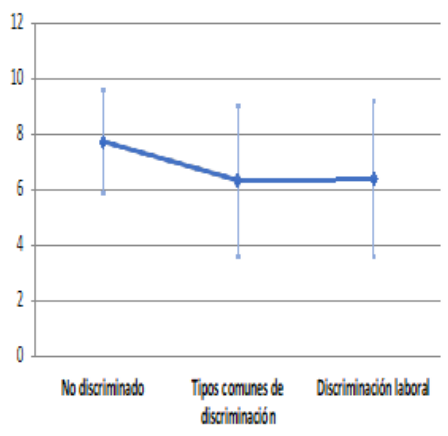

Salud auto-percibida

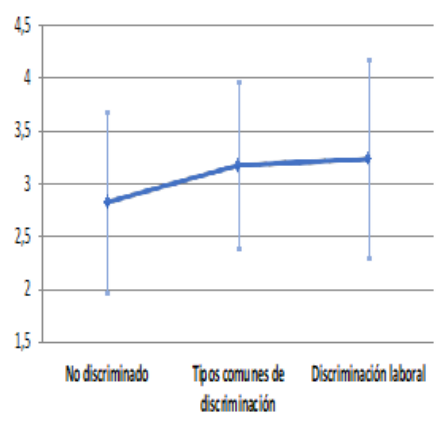

Fuente: Elaboración propia a partir de los datos del Proyecto ANONIMIZADO

En el caso del uso de los servicios de salud, se observa un mayor uso de los servicios de atención primaria entre aquellos que se sienten laboralmente discriminados $(\mathrm{p}<0.05)$, mientras que no se observan diferencias estadísticamente significativas a la hora de acudir a servicios de urgencias, ingresos hospitalarios o especializados. 
Figura 3: Diferencias porcentuales en el uso de servicios de salud según tipo de discriminación percibida

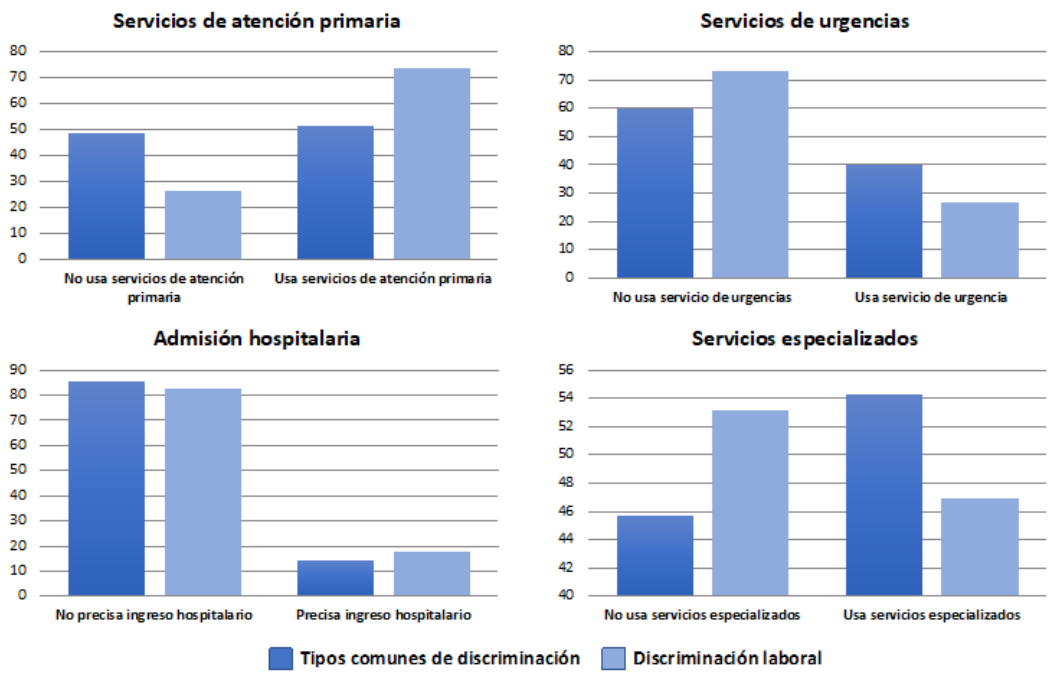

Estos últimos resultados evidencian la asociación negativa de la discriminación laboral y de otros tipos de discriminación sobre la salud. El hecho de que los sujetos discriminados en el ámbito laboral tengan un IMC más elevado (que se asocia con diferentes problemas de salud) y hagan un mayor uso de los servicios sanitarios primarios que los sujetos afectados por otros tipos de discriminación, hace intuir la posibilidad de que quizás la discriminación laboral tenga más efectos nocivos para la salud que el resto de las experiencias discriminatorias examinadas.

La implementación del análisis PSM apoyó parcialmente los resultados obtenidos mediante las técnicas anteriormente aplicadas. Si bien aquellas personas que reportaron discriminación laboral presentaban una mayor propensión a asistir a servicios de atención primaria frente a las personas que se sentían discriminadas por los tipos comunes de discriminación, la técnica PSM no detectó diferencias estadísticamente significativas para la asociación con el IMC. Este dato indica que el análisis ANOVA parece sobreestimar el efecto de la discriminación laboral sobre esta última variable dependiente. Por consiguiente, el análisis confirmó el hecho de que aquellas personas que se han podido sentir laboralmente discriminadas durante los recientes años de crisis económica en Andalucía han asistido con mayor frecuencia al médico de cabecera que aquellos que se sentían discriminados por otras razones.

Finalmente, las puntuaciones de propensión resultantes se utilizaron para comparar gráficamente la probabilidad de uso de servicios de atención primaria (esto es, la variable dependiente que ha arrojado resultados estadísticamente 
significativos mediante la aplicación de dos técnicas diferentes). En la figura 4 se muestran las medidas de propensión resultante de la aplicación de la técnica PSM para la asistencia a servicios de atención primaria por provincias. Estos datos muestran el riesgo poblacional de asistencia a los servicios de atención primaria de aquellas personas que se sienten laboralmente discriminadas frente a las que se sienten discriminadas por otros motivos. Estas medidas ponen de manifiesto que la probabilidad media de acudir al médico de cabecera varía considerablemente entre unas áreas geográficas y otras, encontrándose diferencias por provincias, pero no según ámbito rural o urbano. Salvando el caso de Granada, en el resto de provincias andaluzas la discriminación laboral incrementa el riesgo de que la persona acuda al médico de cabecera frente a las personas discriminadas por otros motivos. Las diferencias en el riesgo de acudir a estos servicios de salud son especialmente acusadas en Almería, Córdoba, Huelva y Sevilla.

Figura 4. Probabilidad de asistir a servicios de atención primaria según el tipo de discriminación percibida por provincias andaluzas.

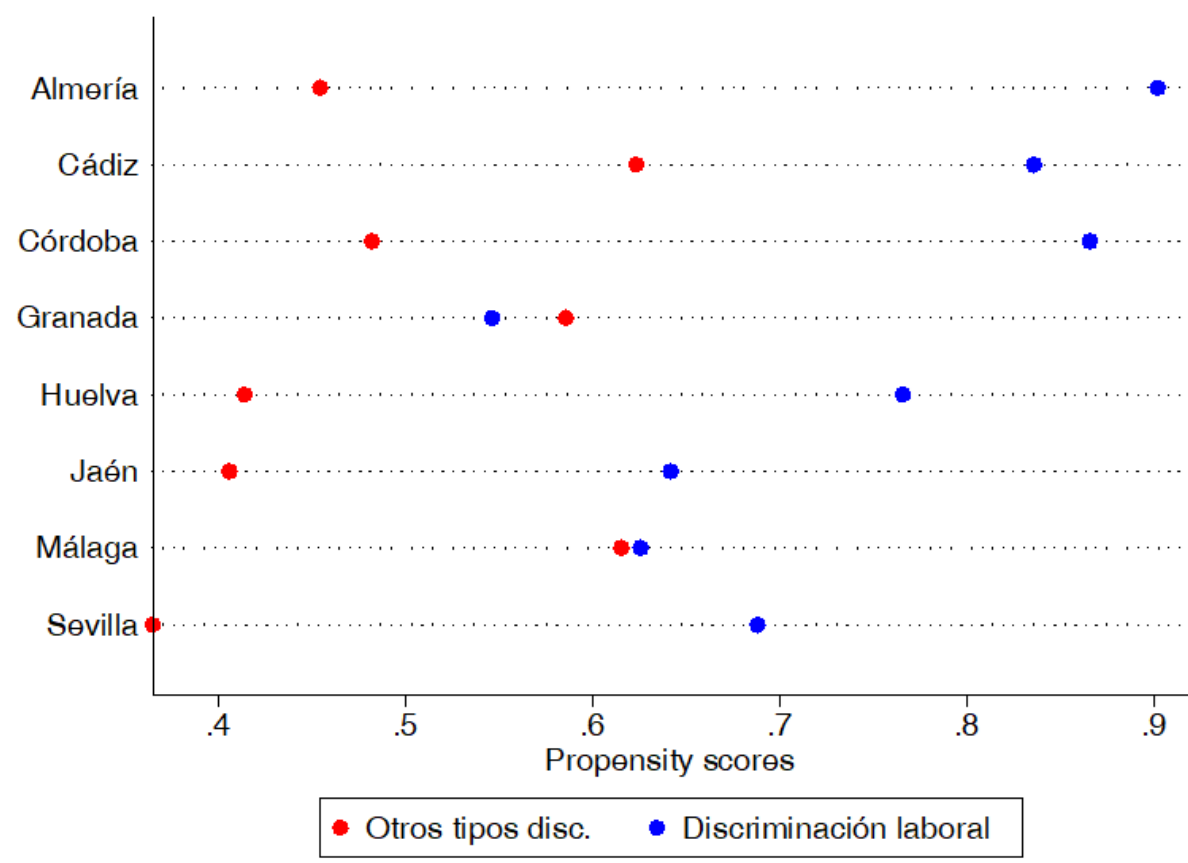

Fuente: Elaboración propia a partir de los datos del Proyecto ANONIMIZADO 


\section{DISCUSIÓN}

Este trabajo de investigación pone de manifiesto que, en el contexto andaluz de los años posteriores a la crisis económica, la discriminación laboral presenta una mayor prevalencia frente a otras formas de discriminación que habitualmente ha sido estudiadas en la literatura. La discriminación laboral es una forma más compleja de discriminación que integra múltiples causas de rechazo, lo que incrementa el riesgo de salud física y mental de aquellas personas que la sufren (Meyer, Schwartz y Frost, 2008; Seng, Lopez, Sperlich, Hamama y Meldrum, 2012).

El 65\% de los entrevistados que reportaban rechazo hacían referencia a otras causas de discriminación, algo atípico teniendo en cuenta que, por término medio, en los países de la UE las personas que reportan otras causas «no habituales» dentro de la investigación en salud rondan el uno y el cinco por ciento según los datos de la Encuesta Social Europea (Alvarez-Galvez y Salvador-Carulla, 2013) . Ahora bien, al analizar los resultados de la pregunta abierta que examinaba esas otras causas de discriminación, lo realmente significativo fue el hecho de que, en Andalucía, la mayor parte de las respuestas hicieran referencia a motivos de discriminación laboral. Unos datos que apuntan a la precaria situación laboral y económica de la población andaluza que no tiene acceso al mercado de trabajo o tiene una situación de riesgo dentro del mismo, al mismo tiempo que se evidencia el carácter complejo de las formas de discriminación laboral mediante la integración de dimensiones de género, edad o discapacidad que afectan al plano laboral y económico.

La asociación de la discriminación laboral con otros tipos de discriminación puede observarse en las respuestas a partir de las que se construye el indicador de discriminación laboral empleado en este estudio. Por ejemplo: mujer en edad fértil que no pueden encontrar un trabajo, falta de oportunidades para grupos jóvenes, ser despedida después de una baja por enfermedad o no encuentra trabajo por ser viejo. Esta información refuerza la idea expuesta anteriormente, de que son los grupos que parten de una posición desfavorecedora aquellos más proclives a experimentar situaciones de discriminación laboral.

El hecho de que los hombres declaren sufrir discriminación laboral en mayor medida que las mujeres no deja de ser paradójico, puesto que no se corresponde con los abundantes hallazgos empíricos que evidencian que las mujeres tienen una peor posición que los hombres en el mercado laboral. No obstante, esta diferencia podría apuntar a "normalización" de este tipo de discriminación entre las mujeres, lo cual no haría sino resaltar la gravedad de las diferencias de género en el mercado laboral andaluz (Alvarez-Galvez et al., 2019). En esta misma línea, se observa que son los grupos en edad laboral son los que presentan porcentajes más elevados de discriminación (es decir, aquellos que ven bloqueado su acceso al mercado de trabajo y por consiguiente sus oportunidades), pero no se encuentran diferencias entre poblaciones que sufren un mayor riesgo de precariedad y vulnerabilidad laboral como son los jóvenes y los mayores de 45 años (Amber 
y Domingo, 2017; García y Echaves, 2017). Por consiguiente, será necesario avanzar en esta línea en futuros estudios.

La tendencia que muestra la discriminación laboral a asociarse con otros tipos de discriminación que también inciden de forma negativa en la salud provoca que una serie de perfiles sociales determinados se encuentren en una situación de especial vulnerabilidad en lo que se refiere al estado de salud, como es el caso de las mujeres inmigrantes que se dedican a las labores domésticas (Benito, Girón, Gil y Parés, 2003) o de los sujetos de etnia gitana con bajos recursos socioeconómicos (Ferrer, 2003).

Mediante este trabajo de investigación podemos observar que sentirse discriminado por motivos laborales resulta igualmente nocivo para nuestra salud y calidad de vida que sentirse discriminado por motivos socio-demográficos, étnicos y/o culturales. Podría decirse que tanto el desempleo como el empleo precario conducen del mismo modo a resultados deficientes de salud, a través del efecto indirecto de la discriminación y la estigmatización de grupos que están excluidos de los mercados laborales y la sociedad. Este resultado nos lleva a concluir que el desempleo y las dificultades socioeconómicas pueden afectar a nuestra salud y calidad de vida a través de procesos de exclusión social basados en desigualdades propias del mundo laboral. Por consiguiente, es esperable que los problemas del desempleo y la precariedad laboral en Andalucía puedan tener importantes consecuencias para la salud futura de la población andaluza, así como en otras regiones españolas que también se encuentran afectadas por elevadas tasas de desempleo y/o precariedad laboral (Álvarez-Gálvez et al., 2019).

Se pone de manifiesto una mayor frecuencia en el uso de los servicios de atención primaria entre aquellos que se sienten laboralmente discriminados, un dato que apunta hacia la mayor prevalencia de problemas de salud, tanto de índole física (dada la relación entre ser discriminado en el ámbito laboral y tener un IMC elevado, el cual se asocia con numerosos problemas de salud) como mental (Seng et. al, 2012; Gayman y Barragan 2013; Milner, Page y Lamontagne, 2014), entre los grupos socio-económicamente desfavorecidos que se enfrentan al desempleo y/o al trabajo precario (Milner, Page y Lamontagne, 2014; Bambra y Eikemo, 2018).

Esta asociación entre la discriminación laboral y los peores resultados de salud puede ayudar a comprender cómo la crisis económica, la falta de ayudas y el aumento del desempleo han podido incrementar los problemas de autoestima, insomnio, estrés y trastornos depresivos de los grupos que, en mayor medida, se han visto afectados por los vaivenes económicos de los últimos años. De hecho, recientes trabajos evidencian que los problemas de salud mental asociados a los problemas económicos y laborales han aumentado durante los años de crisis económica (Glonti et. al, 2015), lo que nos lleva a pensar en su posible relación con los procesos de discriminación laboral que en este trabajo se describen. En este sentido, podrían destacarse dos dinámicas diferentes, señaladas por la bibliografía especializada, a través de las cuales la discriminación laboral influye de forma negativa en la salud mental incidiendo de forma significativa sobre el desarrollo de síntomas depresivos o de ansiedad: las situaciones de tensión o estrés 
en el trabajo unidas al miedo a las consecuencias de la crisis y el desempleo asociado a la precarización del mercado laboral (Giorgi, Arcangeli, Mucci y Cupe1li, 2015; McGee y Thompson, 2015; Zuelke et. al, 2018). Para futuros estudios, también debe tenerse en cuenta el posible riesgo que supone la discriminación en el desarrollo de problemas de salud mental que también se encuentran vinculados a un elevado IMC y la obesidad (Alvarez-Gálvez y Gómez-Baya, 2017).

Los resultados obtenidos, que van en línea con consideraciones como la influencia negativa del desempleo y la discriminación laboral sobre la salud destacadas por la bibliografía especializada tanto a nivel nacional (Urbanos-garrido y López-Valcárcel, 2013), como internacional (Dhanani, Beus, y Joseph, 2018), resaltan la precaria situación laboral y económica de muchos andaluces y andaluzas que no tienen acceso al mercado de trabajo o tienen una situación de riesgo dentro del mismo. Asimismo, cabe señalar que la discriminación laboral se presenta como un proceso que se retroalimenta a sí mismo, pudiendo contribuir a cronificar y perpetuar desigualdades en salud entre diferentes grupos sociales, fundamentalmente por dos razones. Por un lado, el hecho de que la discriminación laboral incida de forma negativa sobre la salud mental implica un proceso circular, siendo numerosas las investigaciones que señalan las dificultades laborales (rechazo de los compañeros, trabas en el acceso al empleo etc.) a las que se enfrentan los sujetos con trastornos mentales (Muñoz, Pérez y Guillen, 2009; Sanjuan, 2011). Es decir, si un sujeto desarrolla un trastorno mental a causa de una situación de discriminación laboral, aumentan las posibilidades de que esta situación se incremente por el desarrollo de dicha enfermedad, lo cual puede contribuir a empeorar la sintomatología de esta. Por otro, en coherencia con las teorías que hacen hincapié en las dinámicas de poder en las que se envuelven las situaciones de discriminación, el hecho de que una de las consecuencias de la discriminación laboral sea la precariedad económica, la cual tiende a empeorar la posición social del sujeto afectado, implica que dicho individuo sea aún más proclive a experimentar situaciones de discriminación (que como se ha evidenciado tienen consecuencias negativas para la salud tanto física como mental), que de nuevo seguirían impactando de forma negativa sobre la posición social del mismo.

En definitiva, una situación de riesgo y vulnerabilidad social que, de no ser abordada de un modo integral mediante la implementación de políticas sanitarias, educativas y sociales orientadas a los grupos en riesgo de exclusión social, puede traducirse en futuras desigualdades de salud y, consecuentemente, en mayores problemas socioeconómicos a través de la progresiva acumulación de desigualdades a lo largo del ciclo vital (Darity, 2003).

\subsection{Limitaciones}

Aunque el presente trabajo de investigación se enmarca en un estudio relativamente amplio y en una muestra representativa de la población andaluza, los resultados obtenidos deben de ser interpretados con cautela. Una limitación de este 
estudio, y en general de los estudios que abordan el tema de la discriminación, es el reducido tamaño de la muestra que reportan alguna forma de rechazo. En este sentido, sería conveniente ampliar el tamaño de la muestra en futuros estudios de cara a obtener un mayor poder explicativo y de generalización de los resultados. En cualquier caso, la identificación de diferencias significativas en el uso de servicios de atención primaria a través de los modelos analíticos empleados y su relación con los resultados obtenidos en estudios previos refuerzan la validez y la correcta dirección de nuestro análisis. Particularmente, mediante el empleo la técnica PSM se posibilitó el correcto equilibrado de la distribución de los factores de confusión observados, reduciendo así posibles sesgos asociados al proceso de selección de unidades de análisis y al conjunto de predictores empleados. De este modo, se trató de contrarrestar el posible efecto de sobre/infra-estimación vinculado al bajo tamaño de la muestra.

También debe tenerse en cuenta que no se incluyó el término "discriminación laboral" como pregunta específica del cuestionario. Pese a ello, los resultados de la prueba alfa de Cronbach muestran que el indicador diseñado goza de una gran consistencia, lo que hace pertinente el análisis del mismo. Finalmente, se pone de manifiesto la necesidad de indagar cualitativamente en los motivos de rechazo y en las consecuencias específicas para la salud de la discriminación laboral, y en particular sobre aquellos colectivos que, en mayor medida, pueden experimentar situaciones de vulnerabilidad social (jóvenes, mujeres, mayores de edad, personas con discapacidad, inmigrantes o aquellos que son discriminados por su condición sexual). Todo ello con el objetivo de promover la equidad futura en la salud y la calidad de vida de la población de nuestras sociedades.

\section{REFERENCIAS}

AMBER, D. y DOMINGO, J. (2017): "Desempleo en mayores de 45 años ¿ Un bucle sin salida?", Revista Brasileira de Orientação Profissional, 18(2), pp. 195-207.

ALCAÑIZ, M. (2015): "Sociología de la (s) violencia (s) de género en España. Una propuesta de análisis", Revista Paz y Conflictos, 8(2), pp. 29-51.

ALCOVER, C., RODRÍGUEZ, F., PASTOR, Y., FERNÁNDEZ, J. Y CHAMBEL, M. (2017): "Crisis económica, salud y bienestar en trabajadores con discapacidad", Revista de Psicología del Trabajo y de las Organizaciones, 33(2), pp. 147-155.

ÁLVAREZ-GÁLVEZ, J. (2016): "Measuring the effect of ethnic and non-ethnic discrimination on Europeans self-rated health", International journal of public health, 61(3), pp. 367-374.

ÁLVAREZ-GÁLVEZ, J. y SALVADOR-CARULLA, L. (2013): “Perceived Discrimination and Self-Rated Health in Europe: Evidence from the European Social Survey (2010)", PLOS ONE, 8(9), e74252, 1-10.

ÁLVAREZ-GÁLVEZ, J., y GÓMEZ-BAYA, D. (2017). Socioeconomic context as a moderator in the relationship between body mass index and depression in Europe. Applied Psychology: Health and Well-Being, 9(3), 410-428. 
ÁlVAREZ-GÁlVEZ, J., SUAREZ-LLEDO, V., MARTINEZ-COUSINOU, G., MUNIATEGUI-AZKONA, E., y GONZALEZ-PORTILLO, A. (2019): “The impact of financial crisis and austerity policies in Andalusia, Spain: disentangling the mechanisms of social inequalities in health through the perceptions and experiences of experts and the general population", International journal for equity in health, 18(1), 108.

AUSTIN, P. (2011):“An introduction to propensity score methods for reducing the effects of confounding in observational studies", Multivariate Behav. Res., 46, pp. 399-424. doi:10.1080/00273171.2011.568786.

BAMBRA, C. y EIKEMO, T. (2018): "Insecurity, Unemployment, and Health: A Social Epidemiological Perspective", en.) The Oxford Handbook of Job Loss and Job Search, Oxford, Oxford University Press, pp. 111-125..

BENITO, M., GIRÓN, M., GIL, C. y PARÉS, A. (2003): "Las mujeres inmigrantes en Sevilla: salud y condiciones de trabajo Una revisión desde la medicina social", Revista Española de Investigaciones sociológicas, 104(3), pp. 123-144.

BOYLAN, J, CUNDIFF, J. y MATTHEWS, K. (2018): "Socioeconomic Status and Cardiovascular Responses to Standardized Stressors: A Systematic Review and Meta-Analysis", Psychosomatic medicine, 80(3), pp. 278-293.

CARPIO, C. y GARCÍA-SERRANO, C. (2015): "Efectos de la crisis en la estructura ocupacional y la biografía laboral de la población inmigrante", Migraciones. Revista del Instituto Universitario de Estudios sobre Migraciones, 37, pp. 75-96.

CHUNG, Y. (2001): "Work discrimination and coping strategies: Conceptual frameworks for counseling lesbians, gay and bisexual clients", Career Development Quarterly, 50, pp. 33-44.

CEPEDA, M., BOSTON, R., FARRAR, J. y STROM, B. (2003): “Comparison of logistic regression versus propensity score when the number of events is low and there are multiple confounders", Am. J. Epidemiol., 158, pp. 280-287. doi:10.1093/aje/ kwg115.

CORRIGAN, P. y WATSON, A. (2002): "Understanding the impact of stigma on people with mental illness", World psychiatry, 1(1), pp. 16-20.

DHANANI, L., BEUS, J. y JOSEPH, L. (2018): "Workplace discrimination: A metaanalytic extension, critique, and future research agenda", Personnel Psychology, 71(2), pp. 147-179.

DARITY, JR. (2003): "Employment discrimination, segregation, and health". American Journal of Public Health, 93(2), pp. 226-231.

FERDINAND, A., PARADIES, Y., PERRY, R. y KELAHER, M. (2014): “Aboriginal health promotion through addressing employment discrimination", Australian journal of primary health, 20(4), pp. 384-388.

FERRER, F. (2003):"El estado de salud del pueblo gitano en España: Una revisión de la bibliografía", Gaceta Sanitaria, 17, pp. 02-08.

GARCÍA, A. y ECHAVES, C. (2017): “Jóvenes aún más precarios: crisis económica y desigualdad laboral en España", Cuadernos de Investigación en Juventud, 2, pp. 3352.

GAYMAN. M. y BARRAGAN, J. (2013): "Multiple perceived reasons for major discrimination and depression", Society and Mental Health, 3(3), pp. 203-220.

GIORGI, G., ARCANGELI, G., MUCCI, N. y CUPELLI, V. (2015): "Economic stress in the workplace: the impact of fear of the crisis on mental health", Work, 51(1), pp. 135-142. 
GLONTI. K., GORDEEV, V., GORYAKIN, Y., REEVES, A., STUCKLER, D., MCKEE, M. y ROBERTS, B. (2015): "A systematic review on health resilience to economic crises", PLOS ONE, 10(4), e0123117, 1-22.

IOÉ, C. (2000): "Discriminación de los inmigrantes en el trabajo", Sociedad y Utopía. Revista de Ciencias Sociales, 16, pp. 91-102.

KIM, S-S. y WILLIAMS, D. (2012): "Perceived Discrimination and Self-Rated Health in South Korea: A Nationally Representative Survey”, PLOS ONE, 7(1), e30501, 1-8.

LAPARRA, M., OBRADORS, A., PÉREZ, B., YRUELA, M., RENES, V., SARASA, S. y TRUJILLO, M. (2007): "Una propuesta de consenso sobre el concepto de exclusión. Implicaciones metodológicas”, Revista española del tercer sector, (5), pp. 15-57.

LIGHTMAN, N. y GOOD, L. (2018): "Measuring economic exclusion for racialized minorities, immigrants and women in Canada: results from 2000 and 2010", Journal of Poverty, 22(5), 398-420.

LINK, B. y PHELAN, J. (2001): "Conceptualizing stigma”, Annual review of Sociology, 27(1), pp. 363-385.

LINK, B. y PHELAN, J. (2014): “Stigma power”, Social Science \& Medicine, 103, pp. 24-32.

MCGEE, R. y THOMPSON, N. (2015): “Unemployment and depression among emerging adults in 12 states, behavioral risk factor surveillance system, 2010", Preventing chronic disease, 12, pp. 1-11.

MEYER, I., SCHWARTZ, S. y FROST, D. (2008): "Social patterning of stress and coping: Does disadvantaged social statuses confer more stress and fewer coping resources?",Social science \& medicine, 67(3), pp. 368-379.

MILLER, D., MAZZA, J. y ECKERT, T. (2009): "Suicide Prevention Programs in the Schools: A review and public health perspective", School Psychology Review, 38(2), pp. 168-188.

MILNER, A., PAGE, A. y LAMONTAGNE, A. (2014): "Cause and effect in studies on unemployment, mental health and suicide: a meta-analytic and conceptual review", Psychological medicine, 44(5), pp. 909-917.

MÍNGUEZ, A. (2015): "La empleabilidad de los jóvenes en España: Explicando el elevado desempleo juvenil durante la recesión económica", Revista Internacional de Investigación en Ciencias Sociales, 11(1), pp. 3-20.

MUÑOZ M., PÉREZ E, CRESPO M. y GUILLEN A. (2009): Estigma y enfermedad mental: análisis del rechazo social que sufren las personas con enfermedad mental, Madrid, Editorial Complutense.

MUÑOZ, L. y MODROÑO, P. (2012): "La desigualdad de género en las crisis económicas", Investigaciones Feministas, 2, pp. 113-132.

PARADIES, Y. (2006): "A systematic review of empirical research on self-reported racism and health", International journal of epidemiology, 35(4), pp. 888-901.

PASCOE, E. y SMART RICHMAN, L. (2009): "Perceived discrimination and health: a meta-analytic review", Psychological Bulletin, 135, pp. 531-554.

PHELAN, J., LINK, B. y DOVIDIO, J. (2008): “¿Stigma and prejudice: one animal or two?", Social science y medicine, 67(3), pp. 358-367.

POMPILI, M., MANCINELLI, I. y TATARELLI, R. (2003): "Stigma as a cause of suicide", The British Journal of Psychiatry, 183, pp. 173-174.

REISS, F. (2013): "Socioeconomic inequalities and mental health problems in children and adolescents: a systematic review", Social science \& medicine, 90, pp. 24-31. 
ROEHLING, M., ROEHLING, P. y PICHLER, S. (2007): “The relationship between body weight and perceived weight-related employment discrimination: The role of sex and race", Journal of Vocational Behavior, 71(2), pp. 300-318.

SANJUAN, A. (2011): "El estigma de los trastornos mentales: discriminación y exclusión social", Quaderns de Psicologia, 13(2), pp. 7-17.

SANTAMARÍA, E. (2002): "Inmigración y barbarie: la construcción social y política del inmigrante como amenaza", Papers: revista de sociología, 66, pp. 0059-75.

SCHUR, E. (1984): Labeling women deviant: Gender, stigma, and social control, New York, Random House.

SENG, J., LOPEZ, W., SPERLICH, M., HAMAMA. L. y MELDRUM, C. (2012): "Marginalized identities, discrimination burden, and mental health: Empirical exploration of an interpersonal-level approach to modeling intersectionality", Social Science \& Medicine, 75(12), pp. 2437-2445.

STRINGHINI, S., CARMELI, C., JOKELA, M., AVENDAÑO, M., MUENNIG, P., GUIDA, F. y CHADEAU-HYAM, M. (2017):"Socioeconomic status and the $25 \times 25$ risk factors as determinants of premature mortality: a multicohort study and metaanalysis of 1. 7 million men and women", The Lancet, 389(10075), pp. 1229-1237.

STUART, H. (2006): "Mental illness and employment discrimination", Current Opinion in Psychiatry, 19(5), pp. 522-526.

STUBER. J., GALEA, S., AHERN, J., BLANEY, S. y FULLER, C. (2003): “The Association between Multiple Domains of Discrimination and Self-assessed Health: A Multilevel Analysis of Latinos and Blacks in Four Low-Income New York City Neighborhoods", Health Services Research, 38(6p2), pp. 1735-1760.

URBANOS-GARRIDO, R. y LÓPEZ-VALCÁRCEL, B. (2013): “Desempleo y salud: un análisis de la repercusión de la crisis económica sobre la salud de los españoles", Estudios de economía aplicada, 31(2), pp. 303-326.

WHELAN, C., LAYTE, R. y MAÎTRE, B. (2003): "Persistent income poverty and deprivation in the European Union: an analysis of the first three waves of the European Community. Household Panel", Journal of Social Policy, 32(1), pp. 1-18.

WILLIAMS, D. y MOHAMMED, S. (2009): "Discrimination and racial disparities in health: evidence and needed research", Journal of behavioral medicine, 32(1), pp. 20-47.

ZUELKE, A., LUCK, T., SCHROETER, M., WITTE, A., HINZ, A., ENGEL, C. y VILLRINGER, A. (2018): "The association between unemployment and depression. Results from the population-based LIFE adult study", Journal of affective disorders, 235, pp. 399-406. 